\title{
Research Article \\ Effect of Dynamic Interaction between microRNA and Transcription Factor on Gene Expression
}

\author{
Qi Zhao, ${ }^{1,2,3}$ Hongsheng Liu, ${ }^{3,4}$ Chenggui Yao, ${ }^{5}$ Jianwei Shuai, ${ }^{1}$ and Xiaoqiang Sun ${ }^{6,7,8}$ \\ ${ }^{1}$ Department of Physics, College of Physics Science and Technology, Xiamen University, Xiamen 361005, China \\ ${ }^{2}$ School of Mathematics, Liaoning University, Shenyang 110036, China \\ ${ }^{3}$ Research Center for Computer Simulating and Information Processing of Bio-Macromolecules of Liaoning Province, \\ Shenyang 110036, China \\ ${ }^{4}$ School of life science, Liaoning University, Shenyang 110036, China \\ ${ }^{5}$ Department of Mathematics, Shaoxing University, Shaoxing 312000, China \\ ${ }^{6}$ Zhongshan School of Medicine, Sun Yat-Sen University, Guangzhou 510080, China \\ ${ }^{7}$ Guangdong Provincial Key Laboratory of Orthopedics and Traumatology, The First Affiliated Hospital of Sun Yat-Sen University, \\ Guangzhou 510000, China \\ ${ }^{8}$ School of Mathematical and Computational Science, Sun Yat-Sen University, Guangzhou 510275, China
}

Correspondence should be addressed to Qi Zhao; zhaoqi@lnu.edu.cn and Xiaoqiang Sun; sunxq6@mail.sysu.edu.cn

Received 11 August 2016; Accepted 10 October 2016

Academic Editor: Huiming Peng

Copyright (C) 2016 Qi Zhao et al. This is an open access article distributed under the Creative Commons Attribution License, which permits unrestricted use, distribution, and reproduction in any medium, provided the original work is properly cited.

\begin{abstract}
MicroRNAs (miRNAs) are endogenous noncoding RNAs which participate in diverse biological processes in animals and plants. They are known to join together with transcription factors and downstream gene, forming a complex and highly interconnected regulatory network. To recognize a few overrepresented motifs which are expected to perform important elementary regulatory functions, we constructed a computational model of miRNA-mediated feedforward loops (FFLs) in which a transcription factor (TF) regulates miRNA and targets gene. Based on the different dynamic interactions between miRNA and TF on gene expression, four possible structural topologies of FFLs with two gate functions (AND gate and OR gate) are introduced. We studied the dynamic behaviors of these different motifs. Furthermore, the relationship between the response time and maximal activation velocity of miRNA was investigated. We found that the curve of response time shows nonmonotonic behavior in Col loop with OR gate. This may help us to infer the mechanism of miRNA binding to the promoter region. At last we investigated the influence of important parameters on the dynamic response of system. We identified that the stationary levels of target gene in all loops were insensitive to the initial value of miRNA.
\end{abstract}

\section{Introduction}

MicroRNAs (miRNAs) $[1,2]$ are a class of endogenous small noncoding RNAs that bind to partially complementary sequences in target mRNAs, negatively regulating their protein production in higher eukaryotes, plants, and animals [1, 3-5]. Many experimental studies have revealed that miRNAs can regulate various biological functions [6, 7], for instance, development and metabolisms [8]. Also, they have been demonstrated to be involved in many cellular signaling regulation processes, including apoptosis, proliferation, and differentiation [9-11]. Moreover, a lot of biological and clinical experiments have shown that miRNAs are involved in the initiation and development of many diseases [12,13], such as cancers [14] and HIV [15]. More and more attention has been focused on the molecular mechanisms related to miRNAs and their functions [16].

The production of miRNA is regulated by certain transcription factors (TFs) that are also key regulators in gene expression. It has been demonstrated that miRNAs and TFs are often highly interacted in a dependent or independent manner [17]. Therefore, miRNA functions can be understood more clearly only in the context of regulatory interactions between TF and miRNA. Experimental data have 


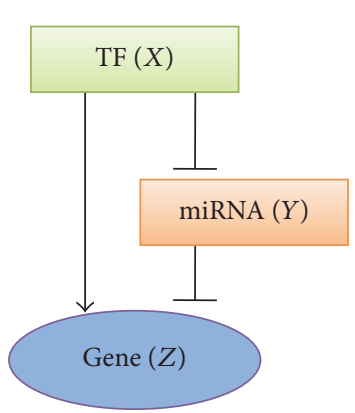

(a)

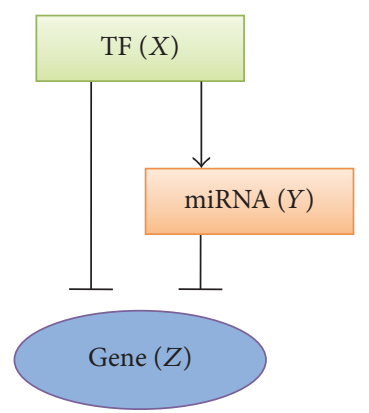

(b)

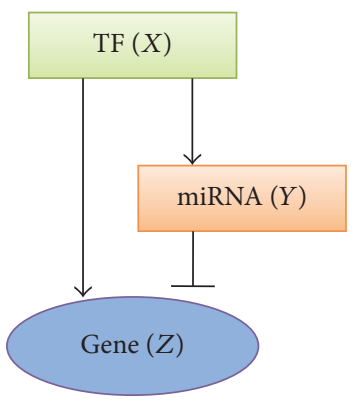

(c)

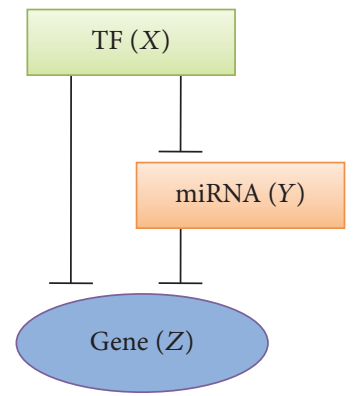

(d)

FIgure 1: The coherent and incoherent feedforward loops. Arrows mean activation, the turned-over T-bars indicate repression. (a) Type 1 coherent FFL, TF activates target gene and represses miRNA synthesis. (b) Type 2 coherent FFL, TF represses target gene and activates miRNA synthesis. (c) Type 1 incoherent FFL, TF activates both target gene and miRNA synthesis. (d) Type 2 incoherent FFL, TF represses both target gene and miRNA synthesis.

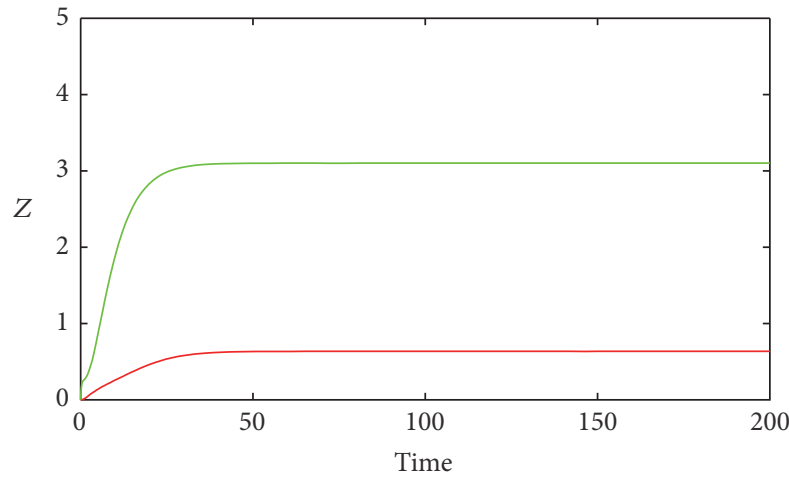

(a)

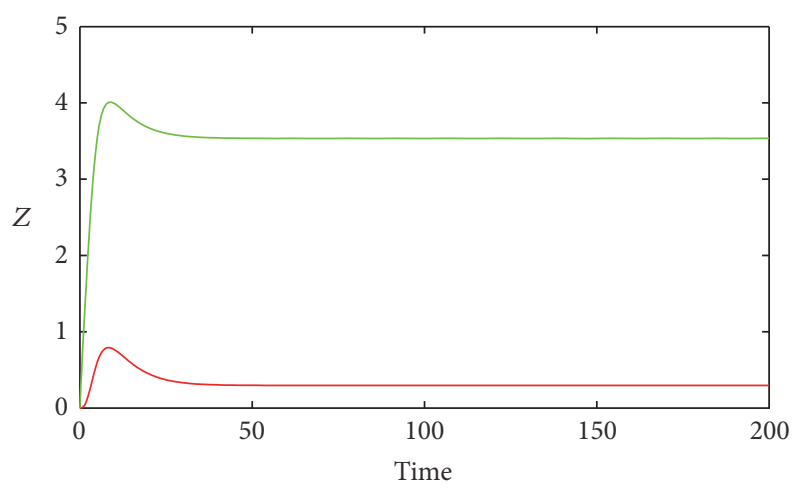

(c)

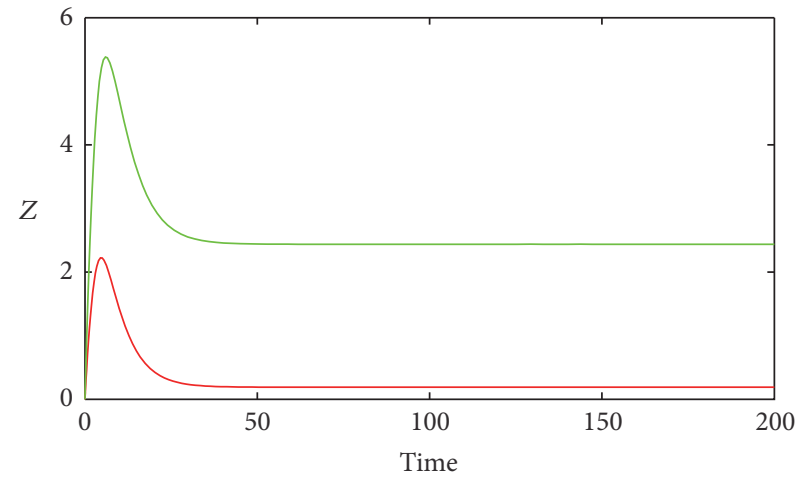

(b)

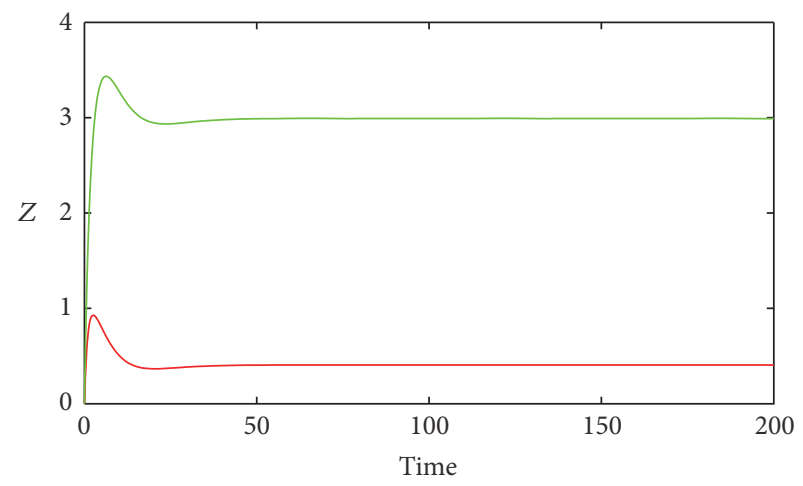

(d)

FIGURE 2: The time evolutions of $Z$ in various FFLs with different gate functions when $k_{1}$ is constant input. Types 1-2 coherent FFLs are shown in (a)-(b), while types 1-2 incoherent FFLs are given in (c)-(d). The red line corresponds to AND gate function, and the green line represents OR gate function. Here we fix $k_{1}=0.25$.

demonstrated that gene regulatory networks are often constituted of some basic subcircuits involving feedforward or feedback loops [18], which are often called motif [19]. Feedforward loops (FFLs) have been shown to be a major member of biological network motifs. Many theoretical works [20-22] and experimental studies [23] have been conducted to investigate their structure and functions within the context of gene expression regulation. These studies focused on FFLs at the transcriptional level, in which gene expression is controlled by two regulatory TFs. Moreover, certain miRNA-containing motifs are often embedded in a lot of gene regulatory networks (GRNs). It has been known that all miRNAs operate through a repressive action on target mRNA. However, considering the interaction between miRNA and TFs, the role of miRNA in gene regulatory network is not simply repressive. Therefore, the investigation 


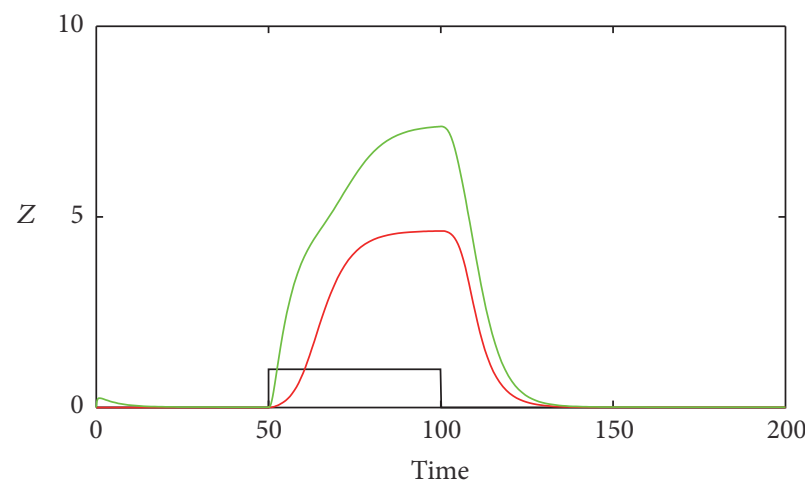

(a)

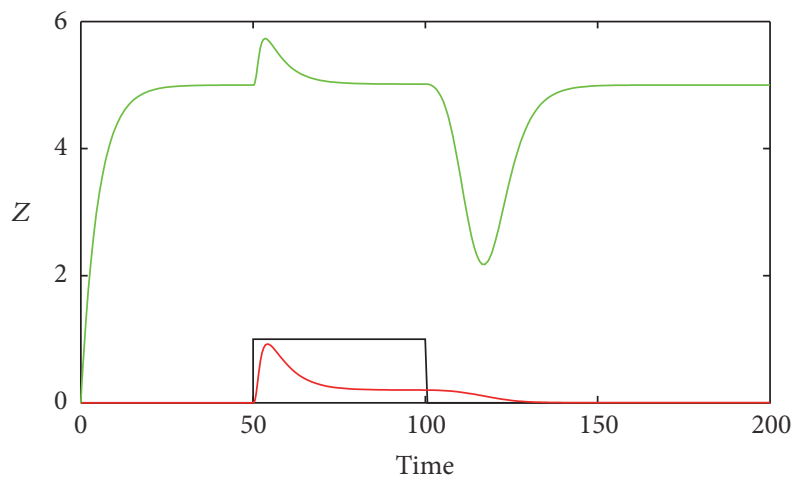

(c)

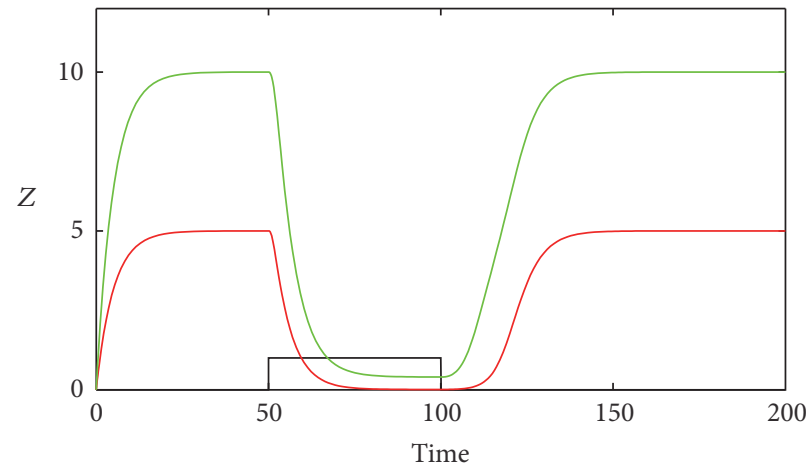

(b)

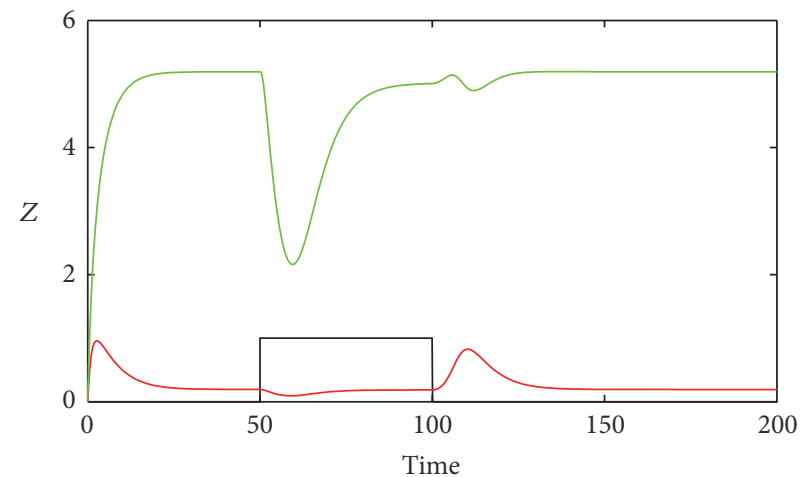

(d)

Figure 3: The time evolutions of $Z$ in various FFLs with different gate functions in response to on and off steps of $k_{1}$. Types 1-2 coherent FFLs are shown in (a)-(b), while types 1-2 incoherent FFLs are given in (c)-(d). The red line corresponds to AND gate function, and the green line represents OR gate function. $k_{1}$ is set to 1 during the time between 50 and 100 and 0 in other time ranges (the black line).

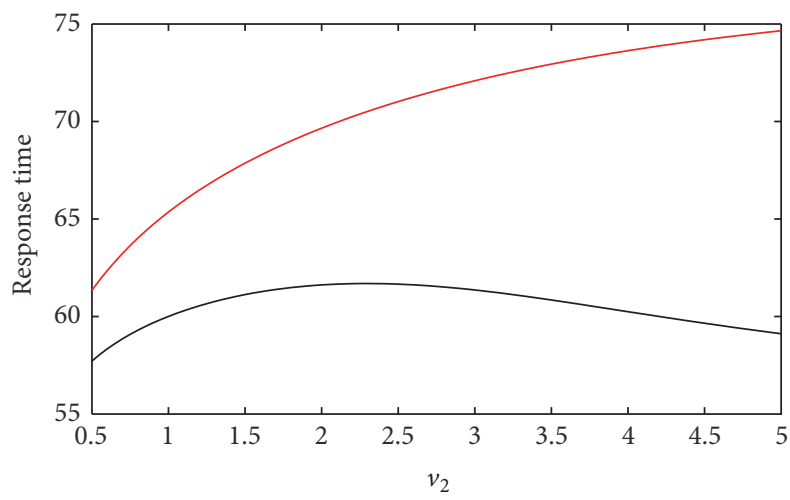

FIGURE 4: The response time is plotted against the variation of $v_{2}$ in Col loop with different gate regulations. The red line corresponds to AND gate function, and the black line represents OR gate function. $k_{1}$ is set to 1 during the time between 50 and 100 and 0 in other time ranges.

of the effect of interaction between TF and miRNA on gene expression is very important to help us understand the role of miRNAs in the GRN and disease.

Mathematical model is a powerful tool used to describe the biological systems and discriminate between different tentative mechanisms [24-36]. Several studies have examined the mechanisms of miRNA-containing motifs using mathematical models. Osella et al. [37] used a detailed analytical model and simulations to investigate the function of the miRNA-mediated FFL. Their analysis demonstrated that the incoherent version of such FFL motif can provide precision and stability to the overall gene expression program with an efficient noise control, given the existence of fluctuations in upstream regulators. Morozova et al. [38] developed a mathematical model containing nine known mechanisms of miRNA action and discriminated among different possible individual mechanisms based on the kinetic signatures. Duk et al. [39] analyzed three mathematical models, in which miRNA either represses translation of its target or promotes target mRNA degradation or is not reused but degrades along with target mRNA. They showed that different mechanisms of miRNA action lead to a variety of types of dynamical behavior of feedforward loops. However, none of previous studies examined the effects of dependence (AND gate) or independence (OR gate) between miRNA and TFs on gene expression.

In this paper, we developed a mathematical model to quantitatively analyze the dynamics of miRNA-containing FFLs and investigate the interaction between miRNA and TF on gene expression. We examined four FFLs, in which each 

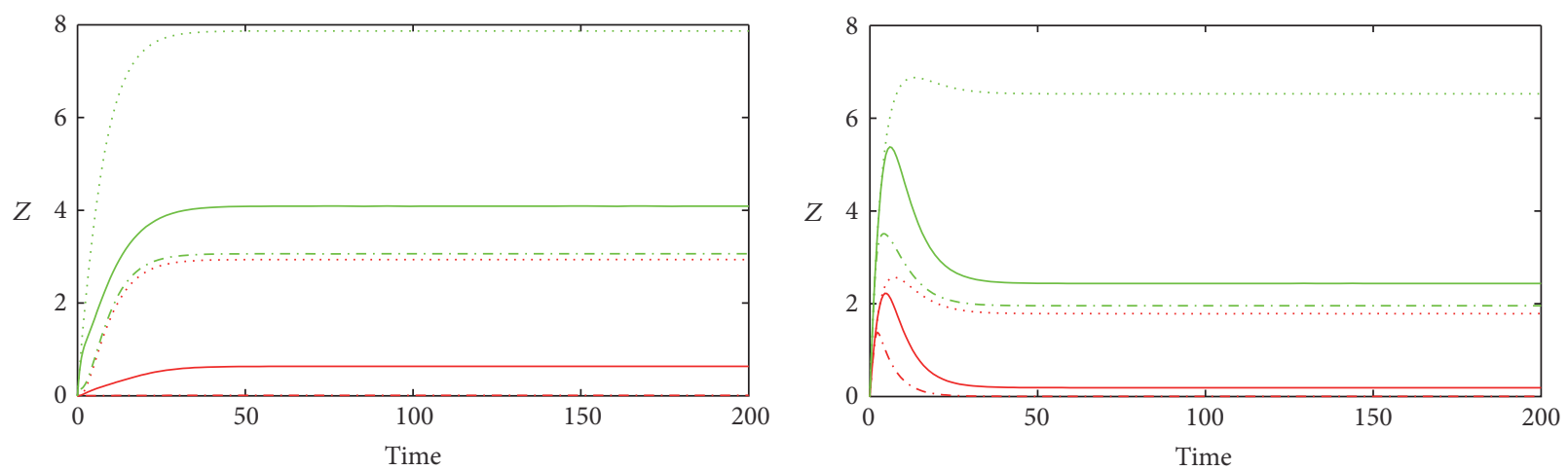

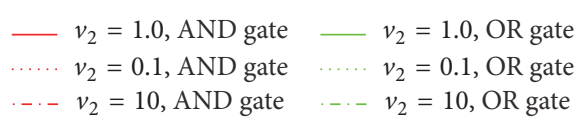

(a)

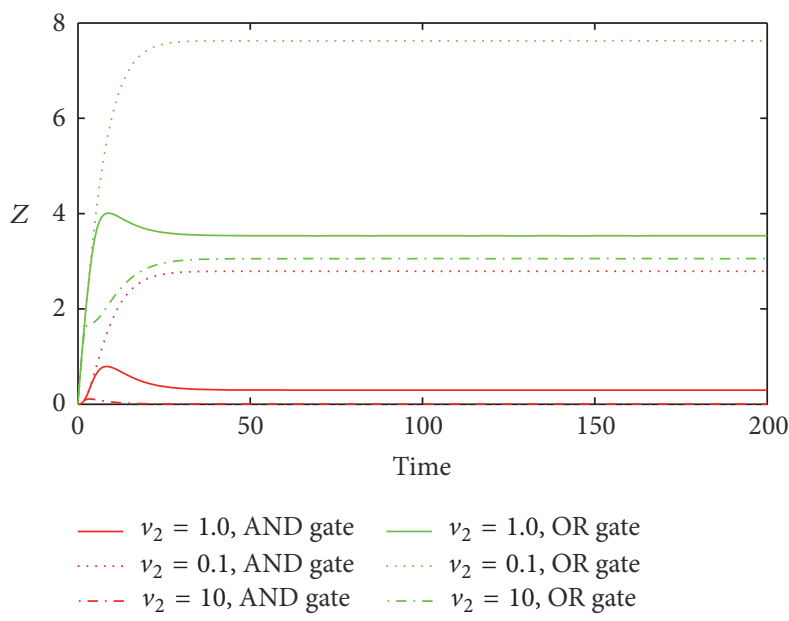

(c)

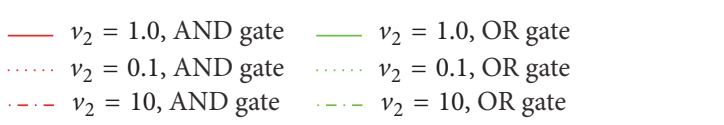

(b)

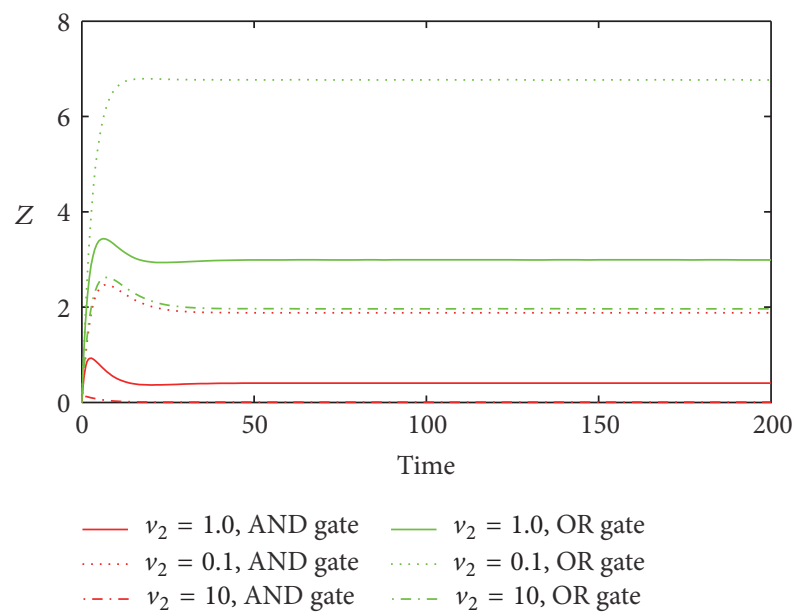

(d)

Figure 5: The time evolutions of $Z$ in various FFLs with different gate functions in response to variation of $v_{2}$. Types 1-2 coherent FFLs are shown in (a)-(b), while types 1-2 incoherent FFLs are given in (c)-(d). The red line corresponds to AND gate function, and the green line represents OR gate function. Here we fix $k_{1}=0.25$.

contains AND gate or OR gate. We analyzed the different dynamical behaviors between AND gate and OR gate for each of these four FFLs. Our results showed that different mechanisms with respect to AND or OR gate might produce distinct dynamics of the GRN. In addition, we examined the relationship between response time of gene expression and certain parameters in the model. Finally we investigated the influence of important parameters on the response of system. Our study advances our quantitative understanding on the dynamic interaction between TF and miRNA, particularly, with AND or OR gate in the GRN, and provides some implications on the miRNA-mediated dieses.

\section{Results}

2.1. Mathematical Model of FFLs. Figure 1 illustrates the general structure of FFLs in miRNA-mediated gene transcription network, similar to that in [24-27]. The upstream transcription factor (TF) regulates the target gene via two parallel pathways: directly and by interaction with miRNA, which also regulates the target gene. Therefore, regulatory interactions in FFL create four possible structural topologies (Figure 1). Two of these configurations are named "coherent": the sign of the direct regulation path from TF to gene is the same as the overall sign of the indirect regulation path from TF via miRNA to gene. The other two structures are termed "incoherent": the sign of the direct regulation path is opposite to that of indirect path. We specify these configurations as type 1 or 2 coherent FFLs and type 1 or 2 incoherent FFLs, respectively. The biological network motif under investigation is described by 3 variables, the concentrations of transcription factor $(X)$, miRNA $(Y)$, and target gene $(Z)$. The dynamical behavior of the FFLs is governed by the following equations:

$$
\frac{d X}{d t}=k_{1}-d_{1} X
$$



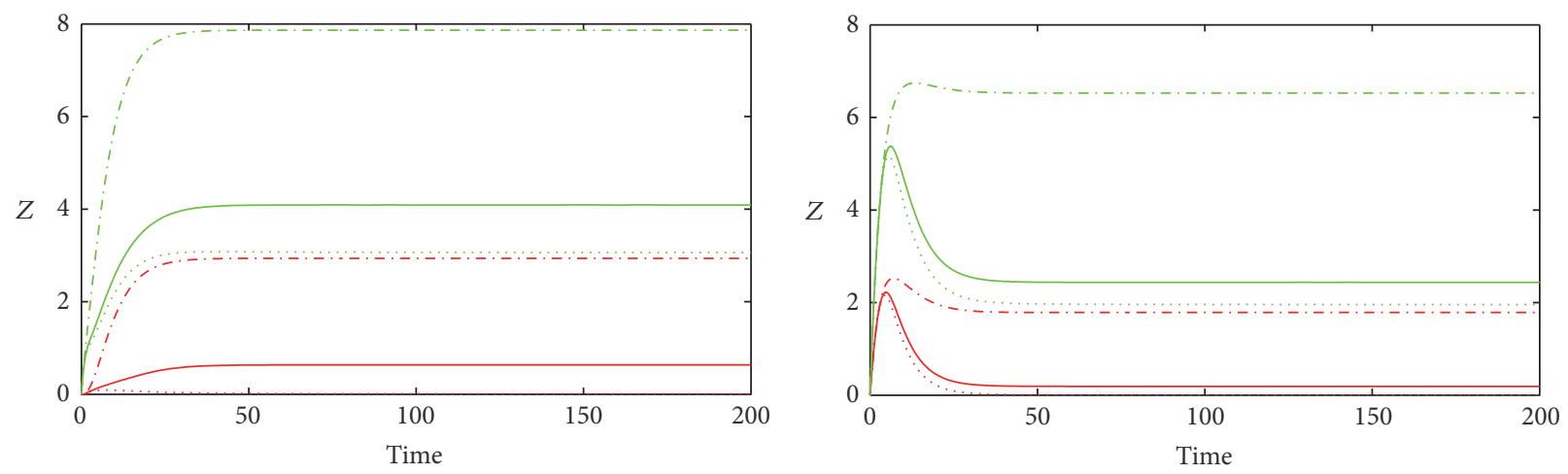

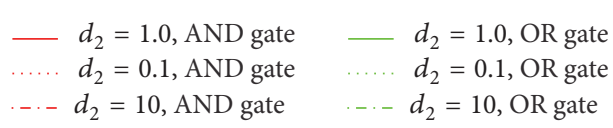

(a)

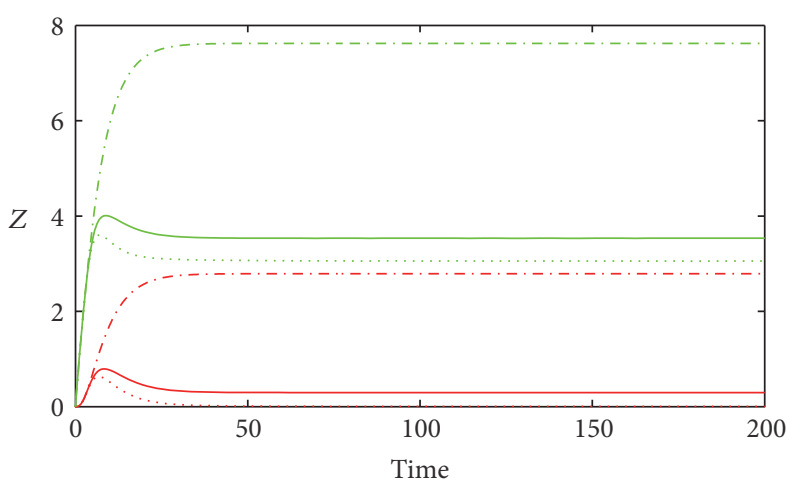

(c)

$$
\begin{aligned}
& -d_{2}=1.0, \text { AND gate }-d_{2}=1.0 \text {, OR gate } \\
& d_{2}=0.1 \text {, AND gate } \ldots . . d_{2}=0.1 \text {, OR gate } \\
& \text {-.. } d_{2}=10 \text {, AND gate }
\end{aligned}
$$

(b)

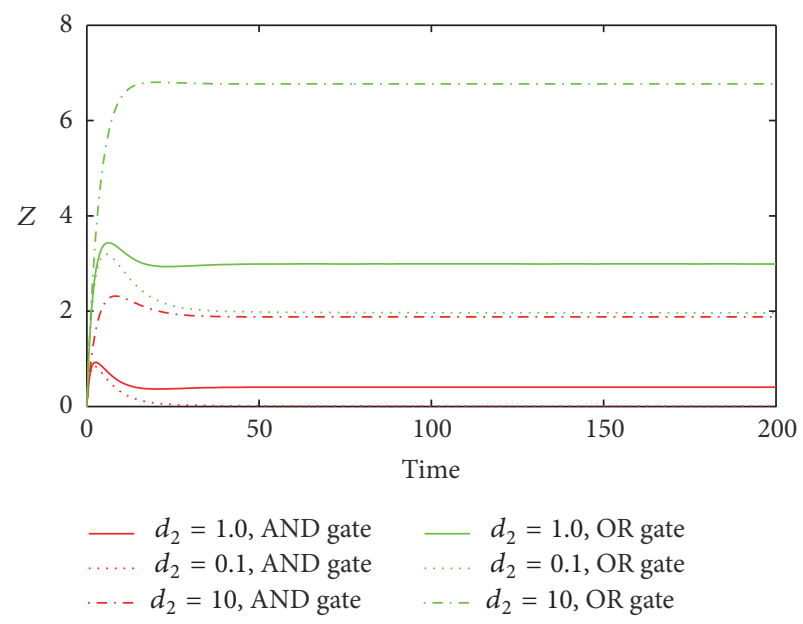

(d)

FIGURE 6: The time evolutions of $Z$ in various FFLs with different gate functions in response to variation of $d_{2}$. Types 1-2 coherent FFLs are shown in (a)-(b), while types 1-2 incoherent FFLs are given in (c)-(d). The red line corresponds to AND gate function, and the green line represents OR gate function. Here we fix $k_{1}=0.25$.

$$
\begin{aligned}
& \frac{d Y}{d t}=v_{2} f\left(X, k_{12}\right)-d_{2} Y, \\
& \frac{d Z}{d t}=v_{3} g\left(X, k_{13} ; Y, k_{23}\right)-d_{3} Z .
\end{aligned}
$$

The regulation function for an activator is $f\left(u, k_{i j}\right)=$ $\left(u / k_{i j}\right)^{n} /\left(1+\left(u / k_{i j}\right)^{n}\right)$ and for a repressor is $f\left(u, k_{i j}\right)=$ $1 /\left(1+\left(u / k_{i j}\right)^{n}\right)$, similar to that we used before in [40, 41]. $g\left(X, k_{13} ; Y, k_{23}\right)$ is the gate function, the mechanisms underlying miRNA-mediated repression are not clear so far, and for this reason we consider that the gate function has two forms. The gate function for an AND gate is $g\left(X, k_{13} ; Y, k_{23}\right)=f\left(X, k_{13}\right) * f\left(Y, k_{23}\right)$, while for an OR gate we have $g\left(X, k_{13} ; Y, k_{23}\right)=f\left(X, k_{13}\right)+f\left(Y, k_{23}\right)$. For more details about the values of parameters and initial concentrations we use, see Tables 1 and 2.
2.2. Comparative Analysis of FFLs' Temporal Behavior under Different Gate Functions. We shall use for brevity the following abbreviations for the FFL identification: Col will mean type 1 coherent FFL, Co2 type 2 coherent FFL, In1 type 1 incoherent FFL, and In 2 type 2 incoherent FFL, respectively.

Figure 2 shows the time courses of $Z$ in various FFLs with different gate functions when $k_{1}$ is constant number. Here $k_{1}$ represents the basal synthesis rate of TF. The dynamics of target gene in Col loop has a form of increasing function and then tends to a constant vale (Figure 2(a)). The target gene profiles in Co2, In1, and In loops show pulse-like behavior due to repression mediated by miRNA (Figures 2(b), 2(c), and $2(d))$. At the steady state, the concentrations of target gene in all the loops with AND gate are much lower than those with OR gate function. It is easy to understand this, because OR gate function makes the synthesis rate bigger than that of AND gate. 
TABLE 1: The values of parameters in the mathematical model.

\begin{tabular}{lccl}
\hline Parameter number & Symbol & Value & Description \\
\hline 1 & $d_{1}$ & 0.2 & Degradation rate of TF \\
2 & $v_{2}$ & 1.0 & Maximal activation velocity of miRNA by TF \\
3 & $d_{2}$ & 0.2 & Degradation rate of miRNA \\
4 & $v_{3}$ & 1.0 & Maximal activation velocity of target gene by TF and miRNA \\
5 & $d_{3}$ & 0.2 & Degradation rate of target gene \\
6 & $k_{12}$ & 1.0 & Michaelis constant of miRNA by TF \\
7 & $k_{13}$ & 1.0 & Michaelis constant of target gene by TF \\
8 & $k_{23}$ & 1.0 & Michaelis constant of target gene by miRNA \\
9 & $n$ & 2 & Hill coefficient \\
\hline
\end{tabular}

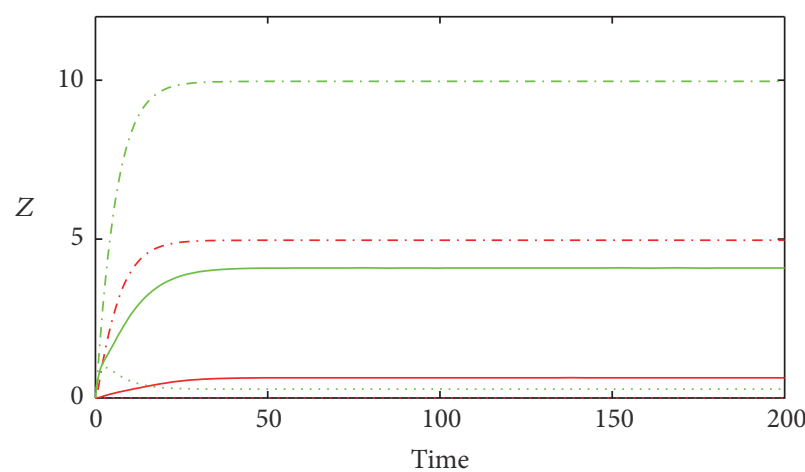

$$
\begin{aligned}
& -k_{1}=1.0, \text { AND gate } \\
& k_{1}=0.1 \text {, AND gate } \\
& \text {-.- } k_{1}=10 \text {, AND gate } \\
& \begin{aligned}
& k_{1}=1.0, \text { OR gate } \\
& \ldots k_{1}=0.1, \text { OR gate } \\
&-k_{1}=10, \text { OR gate }
\end{aligned}
\end{aligned}
$$

(a)

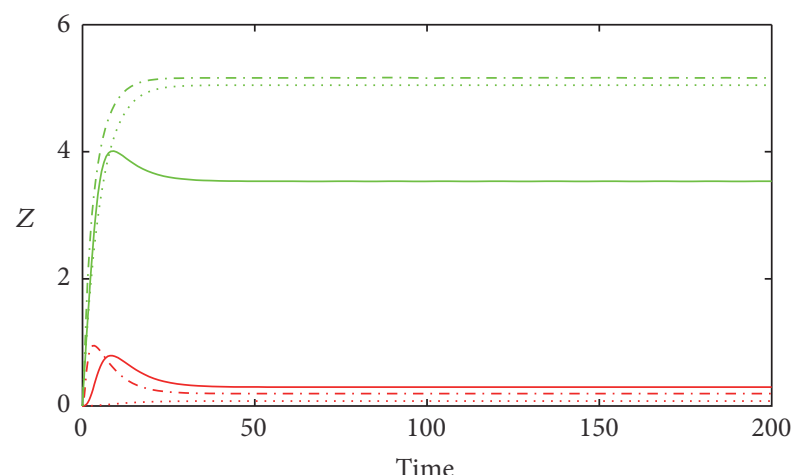

$$
\begin{aligned}
& -k_{1}=1.0 \text {, AND gate } \\
& k_{1}=0.1 \text {, AND gate } \\
& \text {-.. } k_{1}=10 \text {, AND gate } \\
& \begin{aligned}
&-k_{1}=1.0, \text { OR gate } \\
& \ldots \ldots k_{1}=0.1, \text { OR gate } \\
&-.-k_{1}=10, \text { OR gate }
\end{aligned}
\end{aligned}
$$

(c)

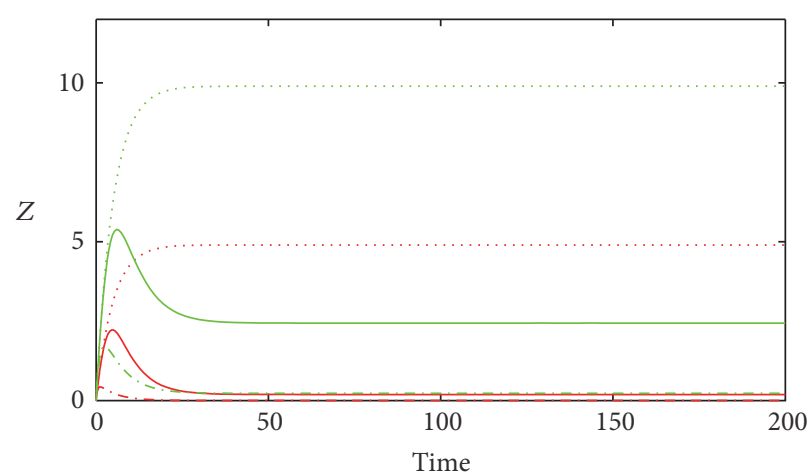

$$
\begin{aligned}
& -k_{1}=1.0, \text { AND gate }-k_{1}=1.0 \text {, OR gate } \\
& k_{1}=0.1 \text {, AND gate } \cdots \cdots k_{1}=0.1 \text {, OR gate } \\
& k_{1}=10 \text {, AND gate } \\
& k_{1}=10, \text { OR gate }
\end{aligned}
$$

(b)

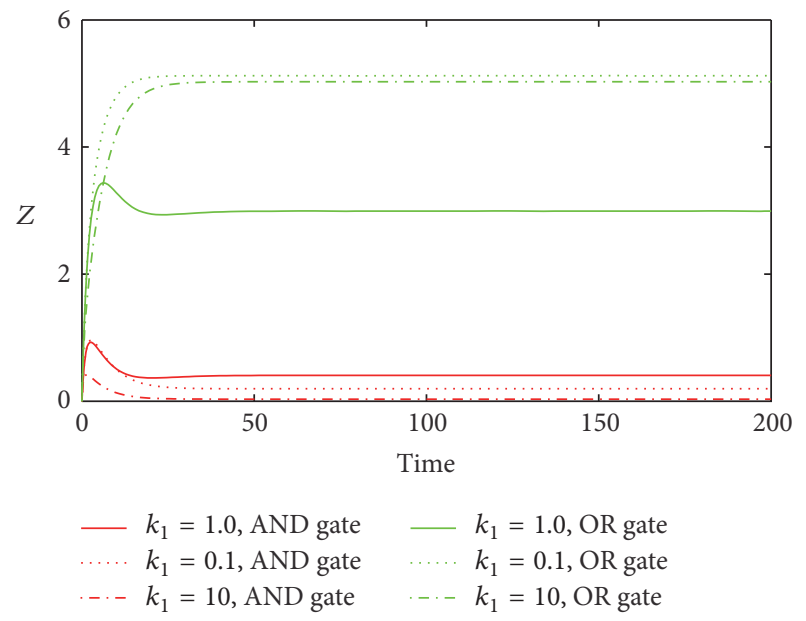

(d)

FIGURE 7: The time evolutions of $Z$ in various FFLs with different gate functions in response to variation of $k_{1}$. Types 1-2 coherent FFLs are shown in (a)-(b), while type 1-2 incoherent FFLs are given in (c)-(d). The red line corresponds to AND gate function, and the green line represents OR gate function. Here we fix $k_{1}=0.25$. 


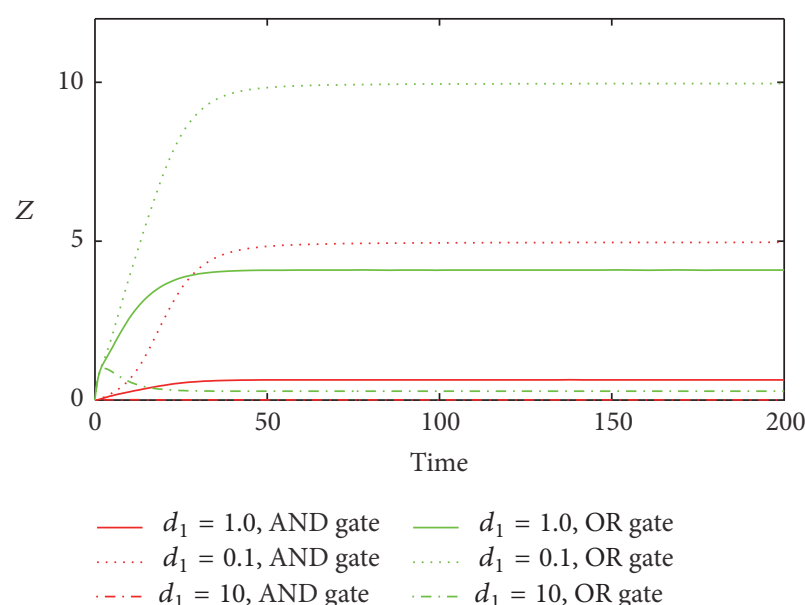

(a)

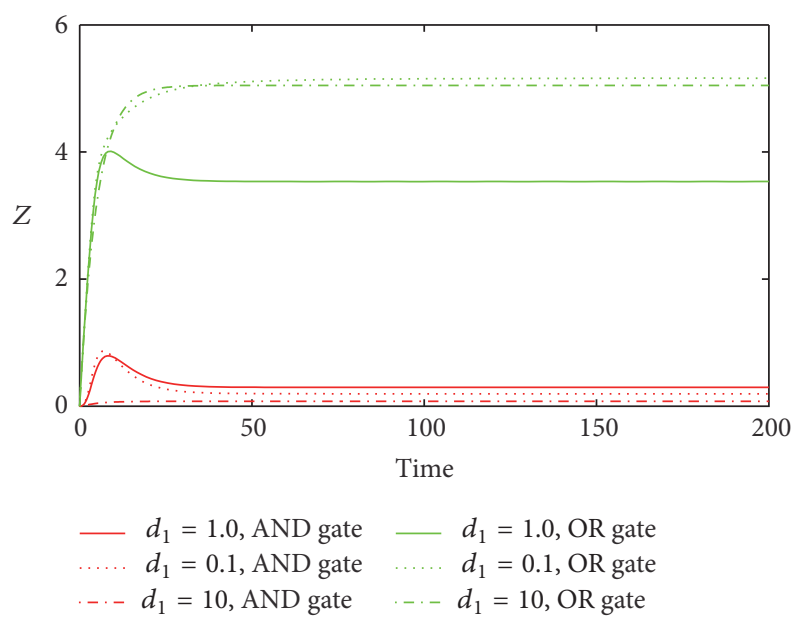

(c)

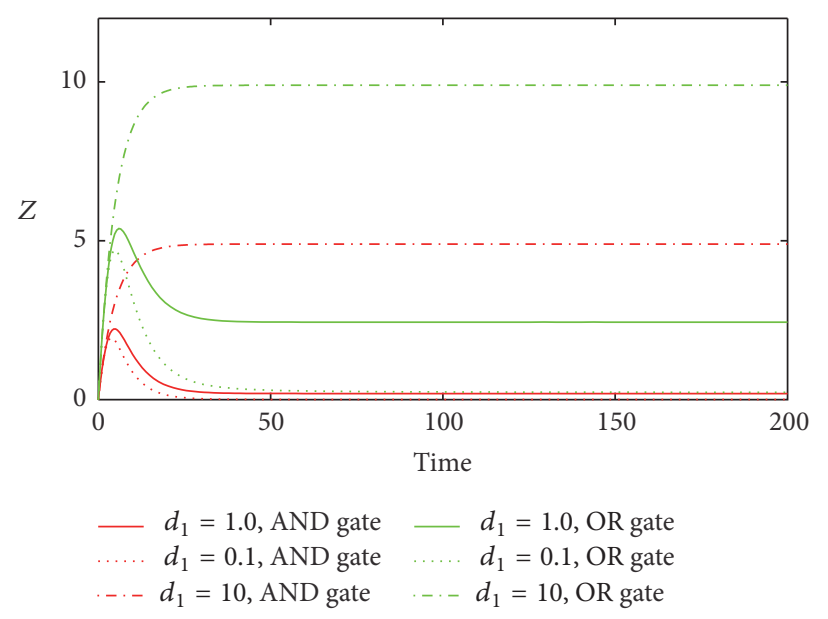

(b)

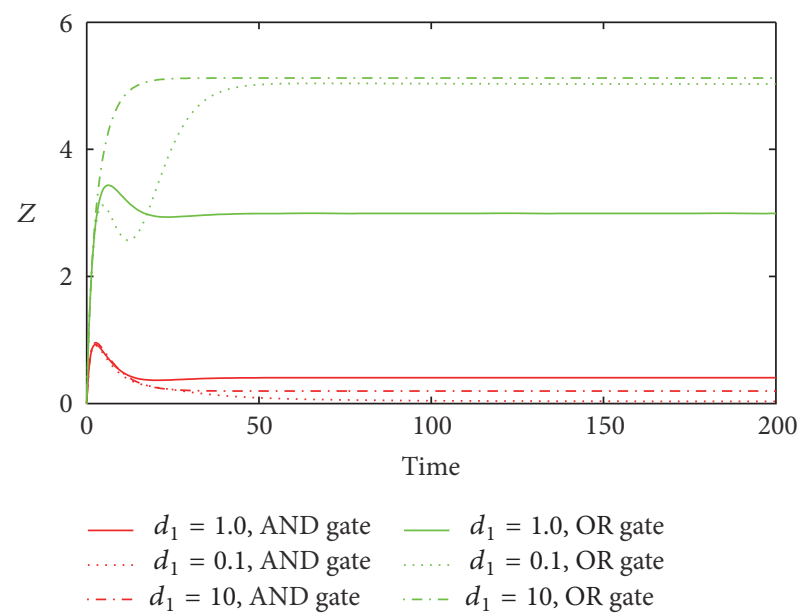

(d)

FIgURE 8: The time evolutions of $Z$ in various FFLs with different gate functions in response to variation of $d_{1}$. Types 1-2 coherent FFLs are shown in (a)-(b), while types 1-2 incoherent FFLs are given in (c)-(d). The red line corresponds to AND gate function, and the green line represents OR gate function. Here we fix $k_{1}=0.25$.

TABLE 2: Initial values of the mathematical model.

\begin{tabular}{lccl}
\hline Parameter number & Symbol & Value & Description \\
\hline 1 & $X$ & 0 & Initial value of TF \\
2 & $Y$ & 0 & Initial value of miRNA \\
3 & $Z$ & 0 & Initial value of target gene \\
\hline
\end{tabular}

Living cells constantly have to respond to a changing environment. To understand how cells deal with a fluctuating environment, we need to know how cells transduce time varying signals. Next we consider the effect of providing the system with simultaneous pulse, a biological scenario which corresponds to continued exposure to environmental stimuli within a certain time range. Accordingly, we set $k_{1}$ to be a piecewise constant function

$$
k_{1}= \begin{cases}1 & 50 \leq t \leq 100 \\ 0 & \text { otherwise }\end{cases}
$$

Figure 3 shows the variations in the response of the output in the motifs. We first compare the kinetics of $Z$ in $\mathrm{Col}$ and Inl loops (Figures 3(a) and 3(c)). When $k_{1}$ turns on, we find out only the steady states of $Z$ in Col and Inl loops with both gate functions rising up due to the direct activation of $Z$ by TF (Figures 3(a) and 3(c)). But in In1 loop, $Z$ first rises slightly and then falls down because TF inhibits $Z$ by promoting miRNA. When $k_{1}$ turns off, both the concentrations of $Z$ in Col and Inl loops decrease, but $Z$ in In1 loop with OR gate eventually grows again to the stationary level. We then compare the kinetics of $Z$ in $\mathrm{Co} 2$ and In 2 loops (Figures 3(b) and $3(\mathrm{~d})$ ); we observe that the concentration of $Z$ in $\mathrm{Co} 2$ loop decreases as $k_{1}$ turns on and increases as $k_{1}$ turns off (Figure 3(b)). But $Z$ in In2 loop with OR gate rises up again to the steady state level after $Z$ falls down, as $k_{1}$ turns on (Figure 3(d)), while $Z$ in In2 loop with AND gate just slightly decreases when $k_{1}$ changes to $1 . Z$ in In 2 loop with two types of gate functions shows pulse-like behavior after $k_{1}$ turns to 0 ; however, the amplitude of $Z$ in In2 loop with OR gate is 

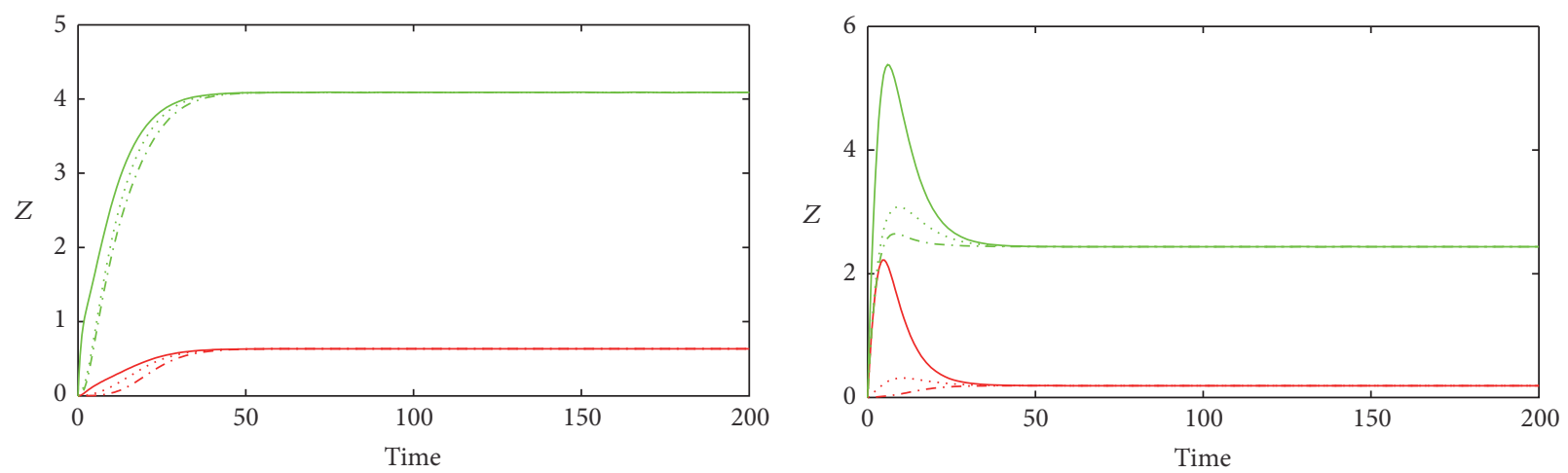

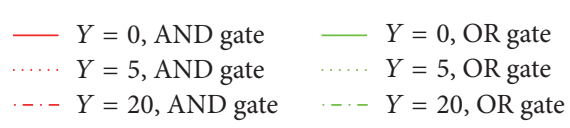

(a)

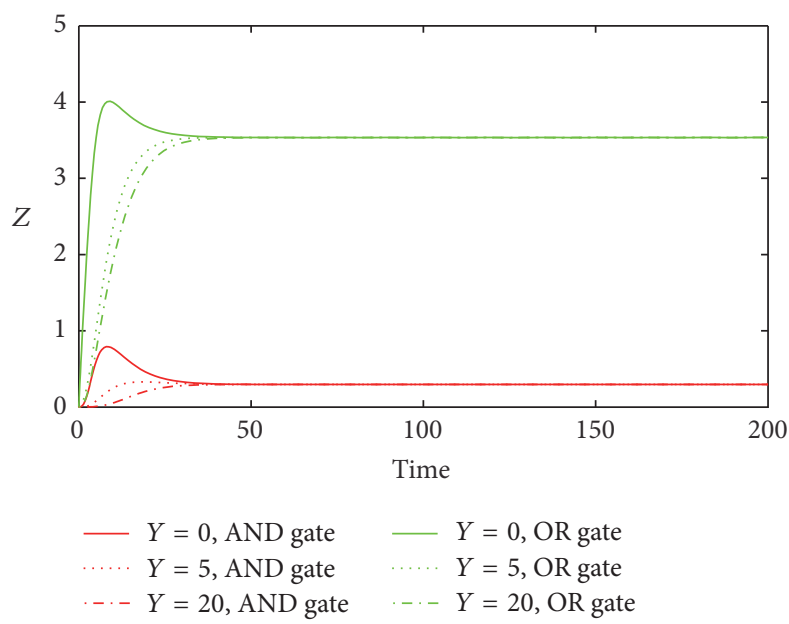

(c)

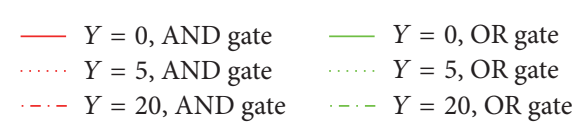

(b)

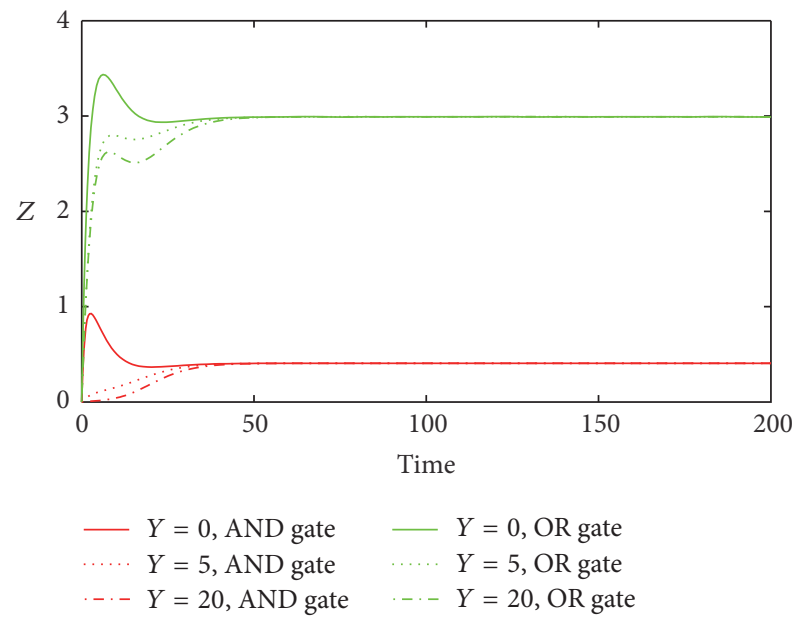

(d)

FIGURE 9: The time evolutions of $Z$ in various FFLs with different gate functions in response to the different initial values of miRNA. Types 1-2 coherent FFLs are shown in (a)-(b), while types 1-2 incoherent FFLs are given in (c)-(d). The red line corresponds to AND gate function, and the green line represents OR gate function. Here we choose three different initial values for $Y, Y=0, Y=5$, and $Y=20$. We fix $k_{1}=0.25$.

much smaller than that with AND gate. From the subfigures in Figure 3, we can find that $Z$ in In2 loop with AND gate is more robust in the presence of $k_{1}$ addition, and $Z$ in In1 loop with AND gate is more stable after an off step of $k_{1}$.

The response time is a measure of the time which a gene product takes to reach its physiologically determined steady state level. The speed of the response is characterized by the response time, which $Z$ takes to reach half of its steady state level. Here $v_{2}$ is the maximal activation velocity of miRNA by TF. In Figure 4, we study the relationship between the response time and $v_{2}$ in Col loop with both gate regulations when providing the system with simultaneous pulse. We can observe that the response time has a form of increasing function as $v_{2}$ turns bigger in Col loop with AND gate, which means the system responses more slowly as $v_{2}$ increases. This is easy to understand; larger $v_{2}$ induces more miRNA generation which further represses target gene synthesis, so the response time turns slowly. But for the case in Col loop with OR gate, the response time shows nonmonotonic behavior, which first climbs and then damps as further increasing $v_{2}$. This indicates that there exists a value of $v_{2}$ such that the system responses most slowly. To understand this, we need to refer to OR gate function we use. It is a nonmonotonic function as $v_{2}$ increases, so the form of function decides the speed of the response of the system. Our result here might be useful to infer the mechanism of miRNA binding to the promoter region, whether or not the TF and miRNA compete for binding to the target gene. Also, we obtain that the response of gene expression in Col loop with OR gate is faster than that in Col loop with AND gate during the period of $v_{2}$ changing.

2.3. Variations of Parameters on the Response of System. It is known that the model coefficients might affect the dynamical behavior of FFLs. Therefore, we further examine how the changes in parameters affect the temporal behavior of the target gene. We investigate the effect of changes in $v_{2}, d_{2}, k_{1}$, and $d_{1}$ on the dynamical behavior of $Z$. 
Figure 5 shows the time course of $Z$ in various FFLs with different gate functions in response to variation of $v_{2}$. We choose three typical values of $v_{2}$ : the original value, 10 fold, and 0.1-fold of $v_{2}$. We find that bigger $v_{2}$ induces less expression of target gene when $Z$ reaches the steady state. We can understand this from the interaction relationship in Figure 1. Larger $v_{2}$ results in more miRNA generation which further represses target gene synthesis, so at last less target gene was observed. Parameter $d_{2}$ is the degradation rate of miRNA. For the influence of $d_{2}$, the situation is opposite, in which bigger $d_{2}$ results in higher level of gene expression after it gets to the stationary level (Figure 6). This is because that larger $d_{2}$ induces less miRNA generation, which results in less inhibition of miRNA on $Z$ synthesis.

We also investigate the effect of changes in $k_{1}$ and $d_{1}$ on the dynamical behavior of $Z$ (Figures 7 and 8). In Col loop, bigger $k_{1}$ induces more $Z$ with both gate functions, while, in Co2 loop, the situation is opposite; lager $k_{1}$ makes less $Z$ with both gate functions. This is due to the fact that TF activates target gene directly and promotes it indirectly in Co1 loop, while, in Co2 loop, TF inhibits target gene directly and represses it indirectly. For the cases in In1 and In2 loops with OR gate, both lager $k_{1}$ and small $k_{1}$ generate nearly the same stationary level of $Z$ which is higher than what the original value makes. For the cases in In1 and In2 loops with AND gate, both lager $k_{1}$ and small $k_{1}$ induce nearly the same stationary level of $Z$ which is slightly lower than that induced by the original value. For the variations of $d_{1}$ (Figure 8), we get similar results in In1 and In2 loops with both gates, but with the opposite results in Co1 and Co2 loops. Furthermore, we study the effect of different initial values of miRNA on the response of the system (Figure 9). We find that the different initial values of miRNA have no significant influence on the steady state of target gene after it passes the transient state.

\section{Conclusions}

In summary, there are multiple variations of the feedforward loops occurring in the nature based on different types of feedback. Hence, we constructed a mathematical model of FFLs in miRNA-mediated gene transcription network. We introduced four possible structural topologies of FFLs associated with two different gate functions which describe the dynamic interaction between miRNA and TF on gene expression. Dynamical behaviors of model component were investigated by computational simulation. Furthermore, the different features of system's response to simultaneous pulse were investigated. The influence of important parameters on the response of system was also considered. We first identified that only the dynamics of target gene in Col loop does not show pulse-like behavior when the synthesis rate of TF is constant. While providing the system with simultaneous pulse, we found that target gene in In2 loop with AND gate is more robust in the presence of stimulus addition, and target gene in Inl loop with AND gate is more stable after an off step of stimulus. Furthermore, we studied the relationship between the response time and maximal activation velocity of miRNA when providing the system with simultaneous pulse. We found that the curve of response time shows nonmonotonic behavior in Col loop with OR gate. We further showed that the stationary levels of target gene in all loops were insensitive to the initial value of miRNA.

\section{Competing Interests}

The authors declare that they have no competing interests.

\section{Authors' Contributions}

Qi Zhao and Xiaoqiang Sun conceived the study, built the model, performed the simulations, interpreted the results, and wrote the paper. Hongsheng Liu and Chenggui Yao participated in discussions. Jianwei Shuai improved and revised the manuscript. All authors have read and approved the final version of the manuscript.

\section{Acknowledgments}

The researches of Qi Zhao and Hongsheng Liu were supported by Innovation Team Project (no. LT2015011) from the Education Department of Liaoning Province. The research of Chenggui Yao was supported by grants from National Natural Science Foundation of China (11675112) and Natural Science Foundation of Zhejiang Province (LY16A050001). The research of Jianwei Shuai was supported by grants from National Natural Science Foundation of China (31370830). The research of Xiaoqiang Sun was supported by grants from National Natural Science Foundation of China (61503419), Guangdong Nature Science Foundation (2014A030310355 and 2016A030313234), the fund for Guangdong Provincial Key Laboratory of Orthopedics and Traumatology (2016B030301002), and "985 project" of Sun Yat-Sen University (no. 50000-31101302).

\section{References}

[1] D. P. Bartel, "MicroRNAs: target recognition and regulatory functions," Cell, vol. 136, no. 2, pp. 215-233, 2009.

[2] L. He and G. J. Hannon, "MicroRNAs: small RNAs with a big role in gene regulation," Nature Reviews Genetics, vol. 5, no. 7, pp. 522-531, 2004.

[3] D. P. Bartel, "MicroRNAs: genomics, biogenesis, mechanism, and function," Cell, vol. 116, no. 2, pp. 281-297, 2004.

[4] R. C. Friedman, K. K.-H. Farh, C. B. Burge, and D. P. Bartel, "Most mammalian mRNAs are conserved targets of microRNAs," Genome Research, vol. 19, no. 1, pp. 92-105, 2009.

[5] O. Voinnet, "Origin, biogenesis, and activity of plant MicroRNAs," Cell, vol. 136, no. 4, pp. 669-687, 2009.

[6] X. Yan, H. Liang, T. Deng et al., “The identification of novel targets of miR-16 and characterization of their biological functions in cancer cells," Molecular Cancer, vol. 12, no. 1, pp. 1-11, 2013.

[7] C. B. Santos-Rebouças and M. M. G. Pimentel, "MicroRNAs: macro challenges on understanding human biological functions and neurological diseases," Current Molecular Medicine, vol. 10, no. 8, pp. 692-704, 2010.

[8] J. Krützfeldt, M. N. Poy, and M. Stoffel, "Strategies to determine the biological function of microRNAs," Nature Genetics, vol. 38, no. 1, pp. S14-S19, 2006. 
[9] G. Suffert, G. Malterer, J. Hausser et al., "Kaposi's sarcoma herpesvirus microRNAs target caspase 3 and regulate apoptosis," PLoS Pathogens, vol. 7, no. 12, Article ID e1002405, 2011.

[10] H. Liang, X. Li, L. Wang et al., "MicroRNAs contribute to promyelocyte apoptosis in $\mathrm{As}_{2} \mathrm{O}_{3}$-treated APL cells," Cellular Physiology \& Biochemistry, vol. 32, no. 6, pp. 1818-1829, 2013.

[11] A. Hata and H. Kang, "Functions of the bone morphogenetic protein signaling pathway through microRNAs (review)," International Journal of Molecular Medicine, vol. 35, no. 3, pp. 563$568,2015$.

[12] V. Pileczki, R. Cojocneanu-Petric, M. Maralani, I. B. Neagoe, and R. Sandulescu, "MicroRNAs as regulators of apoptosis mechanisms in cancer," BMJ Clinical Research Journal, vol. 322, no. 7301, pp. 1528-1532, 2016.

[13] S. Donzelli, M. Cioce, P. Muti, S. Strano, Y. Yarden, and G. Blandino, "MicroRNAs: non-coding fine tuners of receptor tyrosine kinase signalling in cancer," Seminars in Cell \& Developmental Biology, vol. 50, pp. 133-142, 2016.

[14] S. L. Yong and A. Dutta, "MicroRNAs in cancer," Annual Review of Pathology: Mechanisms of Disease, vol. 4, pp. 199-227, 2009.

[15] J. Huang, F. Wang, E. Argyris et al., "Cellular microRNAs contribute to HIV-1 latency in resting primary CD4+ T lymphocytes," Nature Medicine, vol. 13, no. 10, pp. 1241-1247, 2007.

[16] R. Schickel, B. Boyerinas, S.-M. Park, and M. E. Peter, "MicroRNAs: key players in the immune system, differentiation, tumorigenesis and cell death," Oncogene, vol. 27, no. 45, pp. 5959-5974, 2008.

[17] N. J. Martinez and A. J. M. Walhout, "The interplay between transcription factors and microRNAs in genome-scale regulatory networks," BioEssays, vol. 31, no. 4, pp. 435-445, 2009.

[18] A. Re, D. Corá, D. Taverna, and M. Caselle, "Genome-wide survey of microRNA-transcription factor feed-forward regulatory circuits in human," Molecular BioSystems, vol. 5, no. 8, pp. 854867, 2009.

[19] S. Kagale, M. G. Links, and K. Rozwadowski, "Genomewide analysis of ethylene-responsive element binding factorassociated amphiphilic repression motif-containing transcriptional regulators in arabidopsis," Plant Physiology, vol. 152, no. 3, pp. 1109-1134, 2010.

[20] B. Ghosh, R. Karmakar, and I. Bose, "Noise characteristics of feed forward loops," Physical Biology, vol. 2, no. 1, pp. 36-45, 2005.

[21] S. Mangan and U. Alon, "Structure and function of the feedforward loop network motif," Proceedings of the National Academy of Sciences of the United States of America, vol. 100, no. 21, pp. 11980-11985, 2003.

[22] S. Mangan, A. Zaslaver, and U. Alon, "The coherent feedforward loop serves as a sign-sensitive delay element in transcription networks," Journal of Molecular Biology, vol. 334, no. 2, pp. 197204, 2003.

[23] S. Mangan, S. Itzkovitz, A. Zaslaver, and U. Alon, "The incoherent feed-forward loop accelerates the response-time of the gal system of Escherichia coli," Journal of Molecular Biology, vol. 356, no. 5, pp. 1073-1081, 2006.

[24] X. Sun, J. Bao, K. C. Nelson, K. C. Li, G. Kulik, and X. Zhou, "Systems modeling of anti-apoptotic pathways in prostate cancer: psychological stress triggers a synergism pattern switch in drug combination therapy," PLoS Computational Biology, vol. 9, no. 12, article e1003358, 2013.

[25] X. Sun, X. Zheng, J. Zhang, T. Zhou, G. Yan, and W. Zhu, "Mathematical modeling reveals a critical role for cyclin D1 dynamics in phenotype switching during glioma differentiation," FEBS Letters, vol. 589, no. 18, pp. 2304-2311, 2015.

[26] X. Sun, J. Bao, and Y. Shao, "Mathematical modeling of therapyinduced cancer drug resistance: connecting cancer mechanisms to population survival rates," Scientific Reports, vol. 6, Article ID 22498, 2016.

[27] X. Sun, H. Xian, S. Tian et al., "A hierarchical mechanism of RIG-I ubiquitination provides sensitivity, robustness and synergy in antiviral immune responses," Scientific Reports, vol. 6, Article ID 29263, 2016.

[28] X. Chen, C. C. Yan, X. Zhang, and Z. You, "Long non-coding RNAs and complex diseases: from experimental results to computational models," Briefings in Bioinformatics, 2016.

[29] X. Chen, Y.-A. Huang, X.-S. Wang, Z.-H. You, and K. C. C. Chan, "FMLNCSIM: fuzzy measure-based lncRNA functional similarity calculation model," Oncotarget, vol. 7, no. 29, pp. 45948-45958, 2016.

[30] Y. A. Huang, X. Chen, Z. You, D. Huang, and K. C. Chan, "ILNCSIM: improved lncRNA functional similarity calculation model," Oncotarget, vol. 7, no. 18, pp. 25902-25914, 2016.

[31] X. Chen, C. C. Yan, X. Zhang et al., "WBSMDA: within and between score for MiRNA-disease association prediction," Scientific Reports, vol. 6, article 21106, 2016.

[32] X. Chen, "KATZLDA: KATZ measure for the lncRNA-disease association prediction," Scientific Reports, vol. 5, Article ID 16840, 2015.

[33] X. Chen, "Predicting lncRNA-disease associations and constructing lncRNA functional similarity network based on the information of miRNA," Scientific Reports, vol. 5, Article ID 13186, 2015.

[34] Z. Zhu, L. Li, Y. Zhang, Y. Yang, and X. Yang, "Comp Map: a reference-based compression program to speed up read mapping to related reference sequences," Bioinformatics, vol. 31, no. 3, pp. 426-428, 2014.

[35] Z. Zhu, Y. Zhang, Z. Ji, S. He, and X. Yang, "High-throughput DNA sequence data compression," Briefings in Bioinformatics, vol. 16, no. 1, Article ID bbt087, pp. 1-15, 2013.

[36] Y. Zhang, L. Li, Y. Yang, X. Yang, S. He, and Z. Zhu, "Lightweight reference-based compression of FASTQ data," BMC Bioinformatics, vol. 16, no. 1, article 188, 2015.

[37] M. Osella, C. Bosia, D. Corá, and M. Caselle, "The role of incoherent microRNA-mediated feedforward loops in noise buffering," PLoS Computational Biology, vol. 7, no. 3, article e1001101, 2011.

[38] N. Morozova, A. Zinovyev, N. Nonne, L.-L. Pritchard, A. N. Gorban, and A. Harel-Bellan, "Kinetic signatures of microRNA modes of action," RNA, vol. 18, no. 9, pp. 1635-1655, 2012.

[39] M. A. Duk, M. G. Samsonova, and A. M. Samsonov, "Dynamics of miRNA driven feed-forward loop depends upon miRNA action mechanisms," BMC Genomics, vol. 15, supplement 12, pp. 1-18, 2014.

[40] Q. Zhao, M. Yi, and Y. Liu, "Spatial distribution and doseresponse relationship for different operation modes in a reaction-diffusion model of the MAPK cascade," Physical Biology, vol. 8, no. 5, article 055004, 2011.

[41] Q. Zhao, C. Yao, J. Tang, and L. Liu, "Study of spatial signal transduction in bistable switches," Frontiers of Physics, vol. 11, no. 5, Article ID 110501, 2016. 

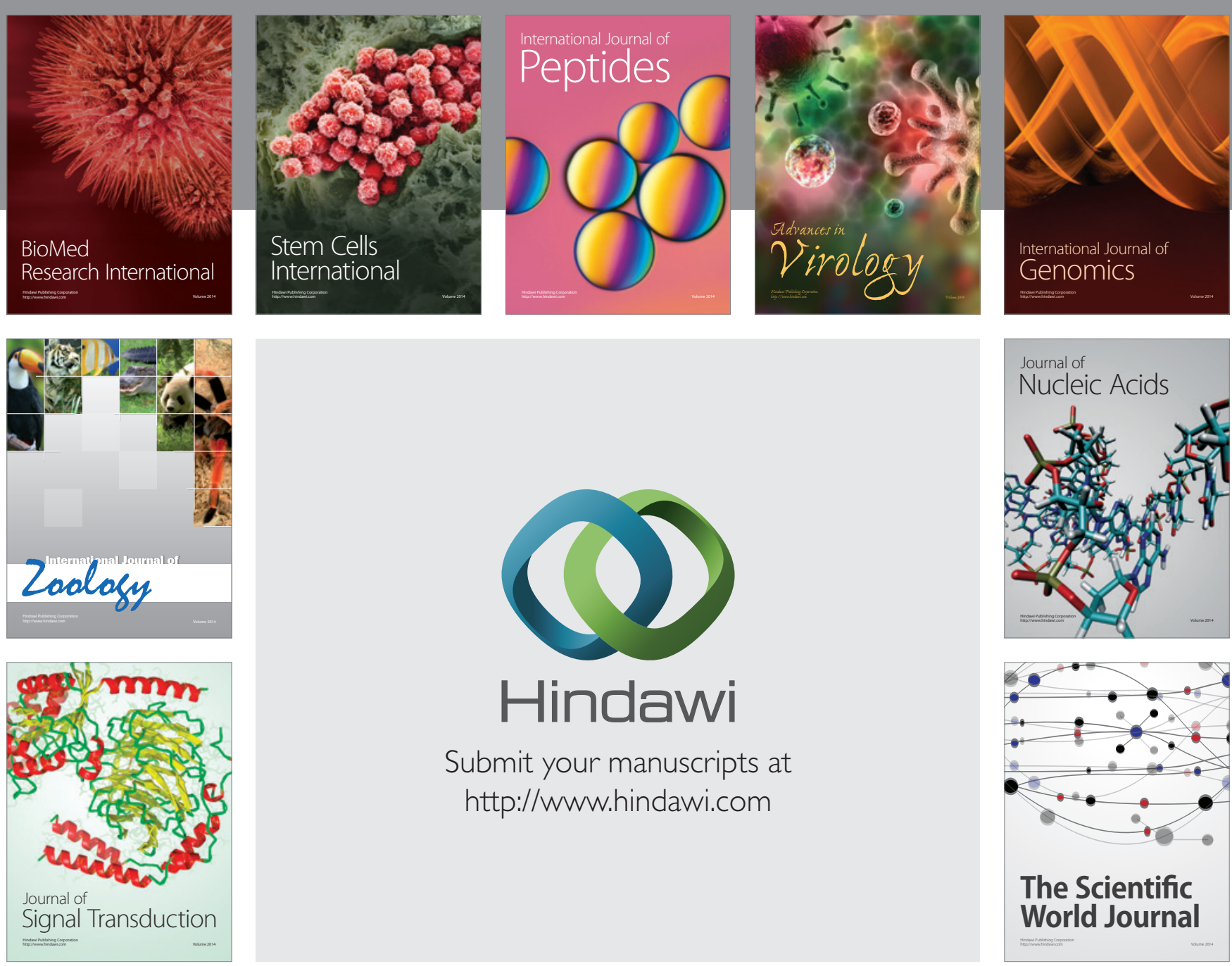

Submit your manuscripts at

http://www.hindawi.com
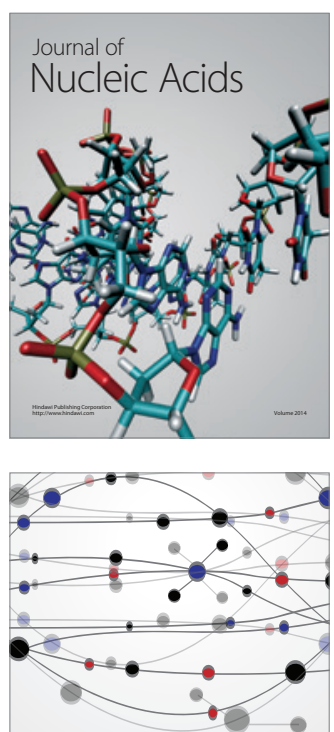

The Scientific World Journal
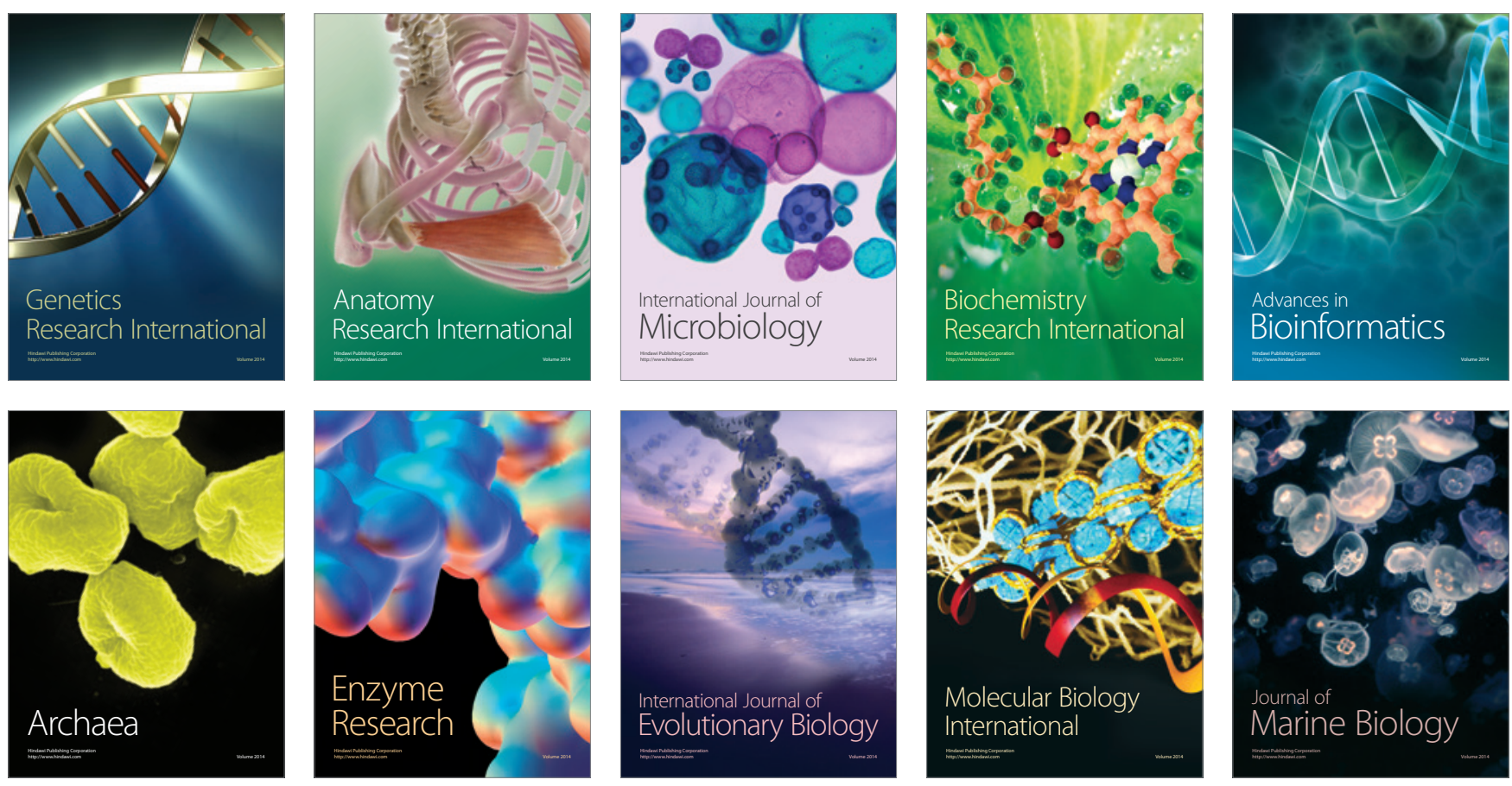